\title{
Detecting magnetic ordering with atomic size electron probes
}

\author{
Juan Carlos Idrobo 1* , Ján Rusz², Jakob Spiegelberg², Michael A. McGuire3 , Christopher T. Symons4, \\ Ranga Raju Vatsavai ${ }^{4}$, Claudia Cantoni ${ }^{3}$ and Andrew R. Lupini ${ }^{3}$
}

\begin{abstract}
Although magnetism originates at the atomic scale, the existing spectroscopic techniques sensitive to magnetic signals only produce spectra with spatial resolution on a larger scale. However, recently, it has been theoretically argued that atomic size electron probes with customized phase distributions can detect magnetic circular dichroism. Here, we report a direct experimental real-space detection of magnetic circular dichroism in aberration-corrected scanning transmission electron microscopy (STEM). Using an atomic size-aberrated electron probe with a customized phase distribution, we reveal the checkerboard antiferromagnetic ordering of Mn moments in LaMnAsO by observing a dichroic signal in the $\mathrm{Mn}$ L-edge. The novel experimental setup presented here, which can easily be implemented in aberration-corrected STEM, opens new paths for probing dichroic signals in materials with unprecedented spatial resolution.
\end{abstract}

Keywords: Aberration correction, STEM, EELS, EMCD, Aberrated probes, Vortex beams

\section{Background}

In the presence of a magnetic field, ferromagnets, paramagnets, antiferromagnets, and ferrimagnets exhibit different photon absorption cross sections that depend on the polarization of the incident photons. This effect, known as dichroism, has allowed polarized X-ray spectroscopy techniques, i.e., X-ray magnetic linear [1] and circular [2] dichroism (XMLD and XMCD), to probe the magnetic properties of materials since the mid-1980s. More recently, it was recognized that in transmission electron microscopy (TEM), under particular scattering conditions, magnetic dichroism could also be probed via energy-loss magnetic circular dichroism (EMCD) spectroscopy [3]. Utilizing crystal as the beam splitter, it was possible to achieve spatial resolutions of about 1-2 $\mathrm{nm}$ $[4,5]$.

Until now, the goal of aberration correction in STEM has been to produce electron probes with sizes as small as possible. The reasoning is that smaller probes result

\footnotetext{
*Correspondence: idrobojc@ornl.gov

1 Oak Ridge National Laboratory, Center for Nanophase Materials

Sciences, Oak Ridge, TN 37831, USA

Full list of author information is available at the end of the article
}

in images and spectra with better spatial resolutions [6-8]. However, it has been recently argued on theoretical grounds that in some cases, it is desirable to have an atomic size electron probe with customized aberrations [9]. According to inelastic electron diffraction calculations, aberrated probes can have tails with a phase distribution that plays an analogous role to polarization of X-rays when interacting with a material. These novel aberrated probes then can be utilized to obtain chiral dichroic signals in materials via electron energy-loss spectroscopy (EELS) with high spatial resolution. Furthermore, the aberrated probes allow the collection of EEL spectra using the transmitted beam, which results in EMCD with intrinsically larger signal-to-noise ratios than those obtained via nanodiffraction techniques [3], where most of the transmitted electrons are discarded.

In this study, we experimentally verify the theoretical prediction of Rusz et al. [9]. We use an aberrated electron probe, which still maintains an atomic size, and detect the antiferromagnetic ordering of Mn moments in LaM$\mathrm{nAsO}$ by observing a dichroic signal in the $\mathrm{Mn} \mathrm{L}$-edge.

EMCD in STEM has been identified previously only in ferromagnets, because the size of the electron probes has been larger than the period over which the magnetic 
moment directions vary in antiferromagnets or ferrimagnets. However, according to Rusz et al. calculations [9], the key to reveal EMCD signals emerging from different magnetic orderings is to set a phase distribution of an atomic size electron probe that maximizes the symmetric component of EMCD at the spectrometer plane. EMCD arising from magnetic moments aligned along the electron beam direction, in a crystal with in-plane fourfold symmetry, can be detected using an aberrated electron probe containing fourfold astigmatism (known as $\mathrm{C}_{34}$ in Krivanek's notation [10], or $A_{3}$ in Haider's notation [11]). Crystals with different symmetries require electron probes with different phase distributions [9]. Here, we use a LaMnAsO as a model system, because it presents an antiferromagnetic ordering at room temperature, which is ideal to test if an aberrated probe can reveal an EMCD signal.

\section{Methods}

\section{Sample preparation and susceptibility measurement}

LaMnAsO has the same room temperature crystal structure as the 1111 family of iron-based superconductors [12], i.e., a layered structure in space group $\mathrm{P} 4 / \mathrm{nmm}$, with lattice constants $a=0.412 \mathrm{~nm}$ and $c=0.903 \mathrm{~nm}$ [13]. The structure is shown schematically in Fig. 1a. LaMnAsO has Mn magnetic moments $\left(\sim 2.4 \mu_{\mathrm{B}}\right.$ at $\left.300 \mathrm{~K}\right)$ aligned antiferromagnetically in a checkerboard pattern in the ab plane, stacked ferromagnetically along the $c$-axis (Fig. 1a). The Néel temperature, $\mathrm{T}_{\mathrm{N}}$, is $360 \mathrm{~K}$, as determined via neutron diffraction experiments [14].

$\mathrm{LaMnAsO}$ was synthesized from LaAs and MnO. An equimolar mixture of the starting materials was mixed and compacted into a pellet. The pellet was sealed inside an evacuated silica glass ampoule and heated at $1000{ }^{\circ} \mathrm{C}$ for $24 \mathrm{~h}$. X-ray diffraction showed the reaction product to be nearly single-phase LaMnAsO, with only $2-3$ wt percent $\mathrm{MnO}$ and $\mathrm{La}_{2} \mathrm{O}_{3}$ detected as impurities. Neither of these phases is expected to influence the magnetic properties near or above room temperature.

More importantly, ferromagnetic $\mathrm{MnAs}\left(T_{\mathrm{C}}=318 \mathrm{~K}\right)$ is not present in the sample, as confirmed by the linear $M$ vs $\mathrm{H}$ data shown in Fig. 1b. Magnetization measurements were performed with a magnetic properties measurement system (Quantum Design). Note that the previous reports of $\mathrm{T}_{\mathrm{N}}$ via magnetization measurements have been masked by the presence of a ferromagnetic MnAs impurity with a Curie temperature of $317 \mathrm{~K}[13,15]$. However, the experimental measurement indicate that no ferromagnetic impurities are present in the synthesized sample, and that the magnetization of one formula unit of LaMnAsO in $2 \mathrm{~T}$ magnetic field is less than $0.2 \%$ of the reported ordered moment per $\mathrm{Mn}$ atom [13]. The low magnetization of LaMnAsO under the presence of
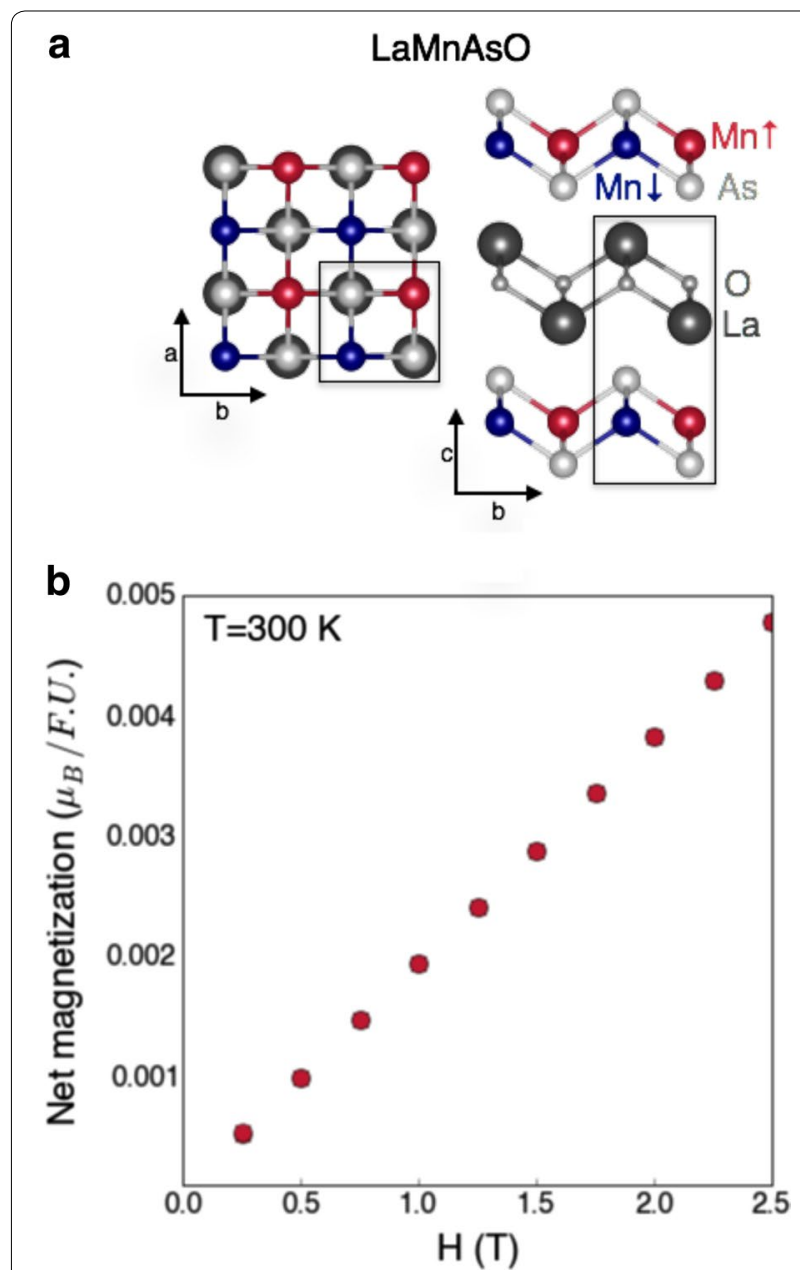

Fig. 1 Crystal structure and magnetic field dependence of the magnetization of $\mathrm{LaMnAsO}$. $\mathbf{a} \mathrm{LaMnAsO}$ has the same structure as the (1111) iron-based superconductors at room temperature [12], i.e., LaFeAsO, but with Mn occupying the Fe sites. The schematic shows the ordering of the Mn magnetic moments, which are antiferromagnetically aligned in a checkerboard pattern in the ab plane, but ferromagnetically aligned along the $c$-axis (i.e., C-type antiferromagnet). The rectangular black boxes outline the unit cell. b Magnetic field dependence of the magnetization of LaMnAsO measured at $300 \mathrm{~K}$ indicates the lack of any ferromagnetic impurity, and minimal canting of the $2.4 \mu_{\mathrm{B}}$ ordered $\mathrm{Mn}$ moments out of the checkerboard state, during the electron microscopy experiments, which are conducted at $\sim 2$ T. F.U. in the vertical axis stands for formula unit

an external magnetic field indicates that its checkerboard antiferromagnetic structure is basically unperturbed during the electron microscopy experiments-which were performed with a field of $\sim 2 \mathrm{~T}$ and at $295 \mathrm{~K}$.

\section{Inelastic electron scattering calculations}

The calculations were performed using a combined multislice/Bloch-waves method [16]. Elastic propagation of an aberrated probe was done by multislice 
calculations, using supercell of size $12 \times 12$ unit cells $\left(4.9 \times 4.9 \mathrm{~nm}^{2}\right)$ in lateral directions and a real-space grid spacing of $6.4 \mathrm{pm}$. The energy-integrated inelastic transition matrix elements were calculated using the method of sum rules inversion [17]. Cut-off parameter in Bloch waves calculations was set to $5 \times 10^{-6}$, and the energy-filtered diffraction patterns were calculated on a grid from $-5 \mathrm{G}$ to $5 \mathrm{G}$ in both directions with a step size of $0.2 \mathrm{G}$, where $G=(100)$. Convergence and collection angles, acceleration voltage were set according to the experiment. The energy-filtered STEM images were evaluated on a grid of $16 \times 16$ pixels within a unit cell. These calculations only obtain the integrated signal of the $\mathrm{L}_{3}$ peak for $3 d$ transition metals. Quenching of the $\mathrm{Mn} \mathrm{L}_{2}$ peak intensity due to multiple scattering effects and effects beyond dipole approximation in inelastic transition matrix elements is not considered.

\section{STEM-EEL spectrum imaging experiments}

The experiments were performed in an aberration-corrected STEM Nion UltraSTEM ${ }^{\mathrm{TM}} 100$, equipped with a cold field emission electron source and a corrector of the third- and fifth-order aberrations, operating with a probe current of $\sim 110 \mathrm{pA}$ at $100 \mathrm{kV}$ accelerating voltage [18]. EEL spectra were collected using a Gatan Enfina spectrometer, with $0.3 \mathrm{eV} /$ channel dispersion, giving an energy resolution of $0.9 \mathrm{eV}$. The convergence semi-angle for the incident probe and the EELS collection semi-angle were $30 \mathrm{mrad}$ and $48 \mathrm{mrad}$, respectively. The sample's thickness was obtained using the log-ratio method with an inelastic mean free path calculated using Lenz' equation as described by Egerton [19]. The EEL spectra data set shown in Fig. $5 b(5 e)$ contains $96 \times 215(50 \times 130)$ spectra, and they were collected with a linear spectral density of $\sim 29.1 \mathrm{spectra} / \mathrm{nm}$ using $20 \mathrm{~ms} /$ pixel dwell time acquisition. The spectra's total acquisition time was from 8 to $13 \mathrm{~min}$. The Z-contrast images were collected using 86-200 mrad half-angle range.

The sample was in the electron microscope at a constant temperature of $22{ }^{\circ} \mathrm{C}(295 \mathrm{~K})$. The temperature of the whole microscope system (both column and room separately) was constantly monitored and controlled with accuracy better than $\pm 0.1{ }^{\circ} \mathrm{C} / \mathrm{h}$. This temperature stability over long periods of time in the electron microscope is one of the main factors that allow the acquisition of spectrum images with atomic resolution. Moreover, the determination of $\mathrm{T}_{N}=360 \mathrm{~K}$ and the negligible net magnetization at room temperature (Fig. 1b) clearly indicates that the electron microscopy experiments were performed within the antiferromagnetic regime. Sample heating due to the electron beam is calculated to be negligible here, considering the thermal conductivity of the material and its low thickness resulting in a small relative amount of inelastically scattered electrons [20].

\section{Evaluation of the signal-to-noise ratio of the measured EMCD spectra}

The EMCD signal-to-noise ratio (SNR) in the measurements was obtained using the following relationship:

$$
\mathrm{SNR}=\operatorname{abs}\left(\Delta L_{2}\right) / \sigma,
$$

where $\Delta \mathrm{L}_{2}$ is the absolute value of the difference of the maximum intensities for the $\mathrm{Mn}_{2} \uparrow$ and $\mathrm{L}_{2} \downarrow$ peaks. $\sigma$ is the total standard deviation of the measurements, which is defined as $\sigma=\sigma \uparrow+\sigma \downarrow$ for correlated noise, and $\sigma=\sqrt{ }\left(\sigma^{2} \uparrow+\sigma^{2} \downarrow\right)$ for uncorrelated noise. $\sigma \uparrow$ and $\sigma \downarrow$ are the standard deviations of the residual signal obtained after performing the background correction of the $\mathrm{Mn} \uparrow$ and $\mathrm{Mn} \downarrow$ spectra before the Mn L-edge onset, respectively. The calculated SNR of the EMCD spectra shown in Fig. $5 \mathrm{f}$ is 9 . The data sets in the performed experiments present an EMCD signature that has an SNR that ranges between 5 and 11 .

\section{Configuring an atomic size fourfold $\left(C_{34}\right)$-aberrated electron probe \\ The $\mathrm{C}_{34}$-aberrated electron probe was achieved using the following approach}

(i) Measure the relative angle of one of the main axes of the corrector with the horizontal direction of the CCD camera.

(ii) Measure the relative angle between one of the two main axes $(a$ or $b)$ of the studied LaMnAsO crystal and the horizontal direction of the CCD camera. This can be obtained by identifying a main Kikuchi line in the sample, for a grain previously aligned in a main zone axis. It is not important, which Kikuchi line is selected in this case. One can select the line that has the smallest angle with respect to the horizontal axis. However, the relative angles of both the corrector and the sample with the horizontal axis need to be defined consistently (either both clockwise or both counterclockwise) [21].

(iii) Calculate the aberration components $\mathrm{C}_{34 \mathrm{a}}$ and $\mathrm{C}_{34 \mathrm{~b}}$ using the measured angle, $\delta$, and the total magnitude of $\mathrm{C}_{34}$ (which for this experiment is defined as $15 \mu \mathrm{m})$, i.e., $C_{34 \mathrm{a}}=\mathrm{C}_{34} \cos \delta, \mathrm{C}_{34 \mathrm{~b}}=\mathrm{C}_{34} \sin \delta$. Here, $\delta$ is the difference of the relative angles between the sample and the corrector with respect to the horizontal axis [21]. The calculated $\mathrm{C}_{34 \mathrm{a}}$ and $\mathrm{C}_{34 \mathrm{~b}}$ values produce the desired antisymmetric phase distribution aligned with the axes of the LaMnAsO crystal as proposed in Ref. 9. 
(iv) Run Nion's Ronchigram aberration correction algorithm [10], using the calculated $\mathrm{C}_{34 \mathrm{a}}$ and $\mathrm{C}_{34 \mathrm{~b}}$ as target values, until reaching convergence within the accuracy of the measurements.

(v) Once the desired aberrations have been added to the electron probe, one can proceed to perform the experiments using any rotation angle in the scanning coils without affecting the measurements.

In a normal operation condition (i.e., used to achieve an aberration-corrected electron probe), Nion's algorithm corrects all the aberrations in the electron optics up to the fifth order as described in Ref. [10]. However, in the setup used here, all the aberrations (up to the fifth order) are corrected with the exception of $\mathrm{C}_{34 \mathrm{a}}$ and $\mathrm{C}_{34 \mathrm{~b}}$, which now have the values obtained in step (ii).

Table 1 displays the values of the third-order aberrations measured by the Nion software for the aberration corrected and the $\mathrm{C}_{34}$-aberrated electron probes used to perform the experiments. We point out that the values presented in Table 1 for $\mathrm{C}_{4} \mathrm{~s}$ and $\mathrm{C}_{5} \mathrm{~s}$ are the same for both probes, because they use previously measured values. The reason is that these higher order aberrations usually remain reasonably constant during long periods of time, and are difficult to measure more accurately.

\section{DFT calculations}

DFT calculations of LaMnAsO were performed using an all-electron full-potential linearized augmented planewaves method implemented in WIEN2k package [22]. Spin-polarized local density approximation (LSDA) was

Table 1 Measured aberrations for the electron probes (shown in Figs. 2, 3) used in the experiments

\begin{tabular}{|c|c|c|}
\hline Aberration & $\begin{array}{l}\text { Corrected probe } \\
\text { Value }\end{array}$ & $\begin{array}{l}\mathrm{C}_{34} \text {-aberrated probe } \\
\text { Value }\end{array}$ \\
\hline$C_{21 a^{\prime}} C_{21 b}$ & $225,129(\mathrm{~nm})$ & $157,196(\mathrm{~nm})$ \\
\hline$C_{23 a^{\prime}} C_{23 b}$ & $-185,44.1(\mathrm{~nm})$ & $-173,48.7(\mathrm{~nm})$ \\
\hline$C_{3}$ & $-0.01(\mu \mathrm{m})$ & $-1.85(\mu \mathrm{m})$ \\
\hline$C_{32 a^{\prime}} C_{32 b}$ & $-1.78,4.22(\mu \mathrm{m})$ & $5.08,9.13(\mu \mathrm{m})$ \\
\hline$C_{34 a^{\prime}} C_{34 b}$ & $0.67,-0.31(\mu \mathrm{m})$ & $9.95,11.5(\mu \mathrm{m})$ \\
\hline $\mathrm{C}_{41 \mathrm{a}^{\prime}} \mathrm{C}_{41 \mathrm{~b}}$ & $-210.7,-262.3(\mu \mathrm{m})$ & $-210.7,-262.3(\mu \mathrm{m})$ \\
\hline $\mathrm{C}_{43 \mathrm{a}^{\prime}} \mathrm{C}_{43 \mathrm{~b}}$ & $348.5,-97.9(\mu \mathrm{m})$ & $348.5,-97.9(\mu \mathrm{m})$ \\
\hline$C_{45 a} C_{45 b}$ & $68.0,-0.03(\mu \mathrm{m})$ & $68.0,-0.03(\mu \mathrm{m})$ \\
\hline$C_{5}$ & $-0.19(\mathrm{~mm})$ & $-0.19(\mathrm{~mm})$ \\
\hline$C_{52 a^{\prime}} C_{52 b}$ & $-1.18,-7.41(\mathrm{~mm})$ & $-1.18,-7.41(\mathrm{~mm})$ \\
\hline$C_{54 a^{\prime}} C_{54 b}$ & $-1.75,-0.14(\mathrm{~mm})$ & $-1.75,-0.14(\mathrm{~mm})$ \\
\hline$C_{56 a^{\prime}} C_{56 b}$ & $-0.63,-0.17(\mathrm{~mm})$ & $-0.63,-0.17(\mathrm{~mm})$ \\
\hline
\end{tabular}

Defocus, $C_{12 a}$ and $C_{12 b}$ were corrected manually during data acquisition. $C_{12} s$ were corrected within few tens of nanometers until the images show the best contrast. Here, the subscripts a and b refer to the optical axes of the corrector used to describe exchange and correlation effects, including a $+\mathrm{U}$ correction (LSDA $+\mathrm{U}$ method) with $U=6 \mathrm{eV}$ and $J=1 \mathrm{eV}$, using the around mean-field (AMF) double-counting correction [23]. Spin-orbital coupling was included in the calculations. A large basis size of more than 120 basis functions per atom and $660 \mathrm{k}$-points in the irreducible wedge of the Brillouin zone were used to ensure a well-converged calculation.

\section{Results and discussion}

Figure $2 \mathrm{a}$ and $\mathrm{b}$ shows the electron probe intensity and phase distribution calculated using the measured values of residual aberrations obtained in an aberration-corrected STEM after all aberrations up to the fifth order have been corrected.

Adding aberrations to the electron probe, such as $\mathrm{C}_{34}$, obviously affect the probe by increasing its full width at half maximum (FWHM) and changing the shape of its tails. However, the calculations done for this work show that for electrons accelerated at $100 \mathrm{kV}, \mathrm{a} \mathrm{C}_{34}$ value of $15 \mu \mathrm{m}$ still maintains the atomic size of the electron probe, and the desired phase distribution on the tails allows measurement of EMCD signals with atomic resolution in LaMnAsO. Figure 2c, d shows the calculated electron probe intensity and phase distribution obtained by correcting all the aberrations up to the fifth order apart from $\mathrm{C}_{34}$, which was set to $15 \mu \mathrm{m}$. The $\mathrm{C}_{34}$ aberrated probe still has an atomic size and shows tails with relative large intensities when compared with the corrected probe, as shown in Fig. 3.

Figure 2e, $\mathrm{f}$ shows the experimental $Z$-contrast STEM images of a LaMnAsO grain oriented along the $c$-axis obtained with the corrected probe and the $\mathrm{C}_{34}$ aberrated probe, respectively. Noting that in the $Z$-contrast image acquired using the $\mathrm{C}_{34}$ aberrated probe (Fig. 2f), it is still possible to resolve individual $\mathrm{La} / \mathrm{As}$ and $\mathrm{Mn} / \mathrm{O}$ atomic columns, although an increase in the background and the $\mathrm{Mn} / \mathrm{O}$ atomic column intensities can be observed when compared with the image acquired with the corrected probe. The increase in background is due to larger tails of the $\mathrm{C}_{34}$ aberrated probe.

In a previous theoretical study [16], it was noted that with electron vortex beams [24-27], an EMCD signal emerges only from probe positions that are close to the center of a magnetic atomic column. The localization of the EMCD signal contrasts to other EELS signals [19], which can, in principle, be non-local [28]. We calculated the effective EMCD signal by taking the ratio between the inelastic scattering cross section evaluated solely using the imaginary part of the mixed dynamical formfactor (which corresponds to the magnetic signal-i.e., $\mathrm{Mn} \uparrow-\mathrm{Mn} \downarrow)$ and the total inelastic scattering cross section arising in the EELS experiments [16]. The calculations were performed with a $29-\mathrm{nm}$ thick $\mathrm{LaMnAsO}$ 

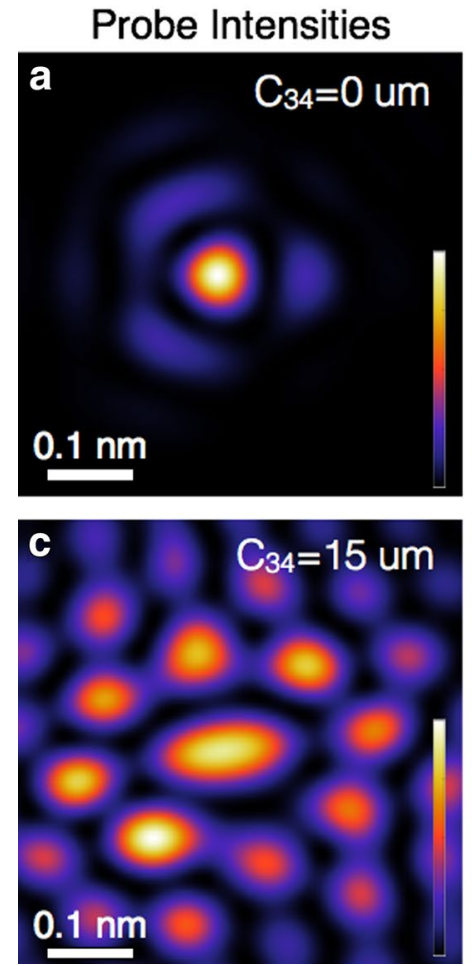
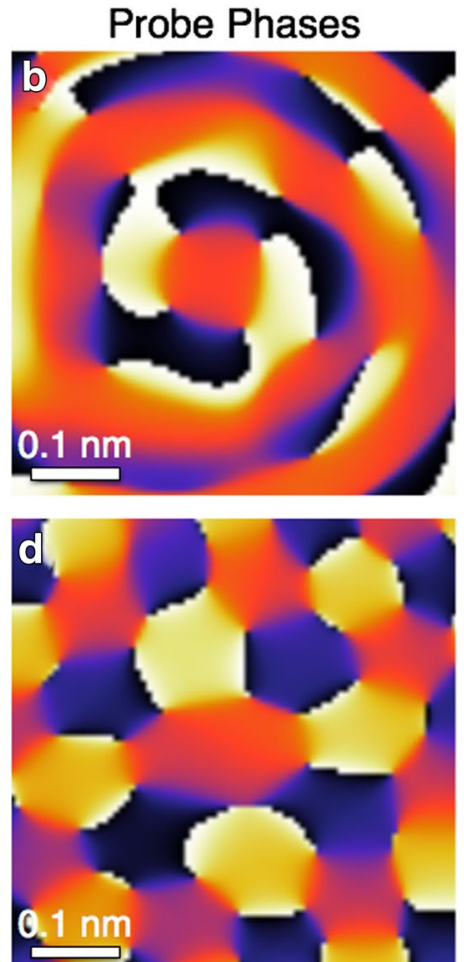
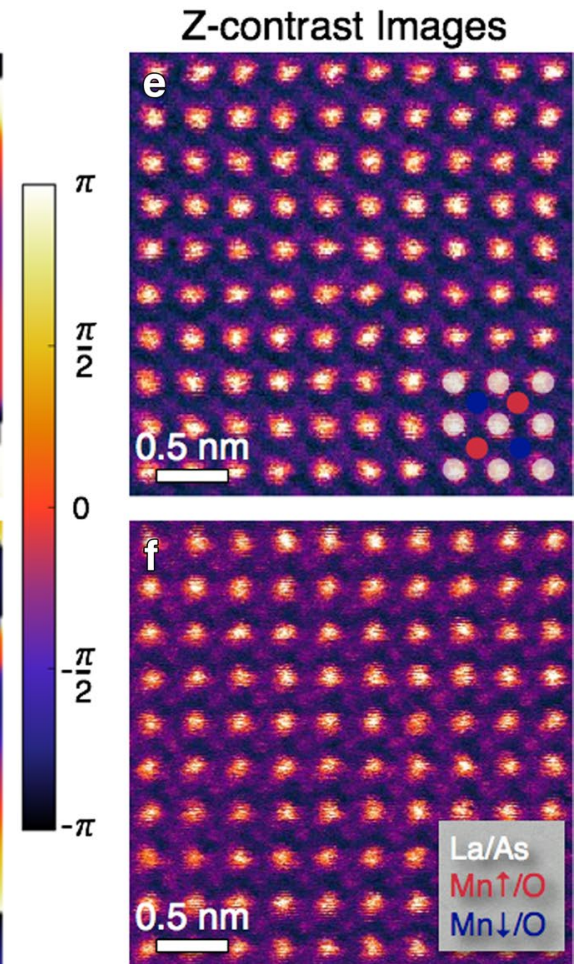

Fig. 2 a Electron probe intensity and $\mathbf{b}$ phase distribution obtained by correcting all the aberrations (up to the fifth order) of the electron optics (corrected probe). The distortion observed in the probe intensity from an ideal Airy-Gaussian-like shape, and in the probe phase from an ideal zero phase, is due to the residual third order $\left(C_{32}\right)$ aberrations. $\mathbf{c}$ Electron probe intensity and $\mathbf{d}$ phase distribution obtained by correcting all the aberrations (up to the fifth order) with the exception of fourfold astigmatism, $C_{34}$, which was explicitly set to $15 \mu \mathrm{m}$. The aberrated electron probe still has an atomic size, but it also shows tails with relative large intensities when compared with the corrected probe. The phase distribution of the tails has a fourfold symmetry that varies from $\pi / 2$ to $-\pi / 2$ between each adjacent tail. $\mathbf{e}, \mathbf{f} Z$-contrast STEM images of a LaMnAsO grain oriented along the $c$-axis and obtained with the corrected probe [shown in (a)] and with the $\mathrm{C}_{34}$ aberrated probe [shown in (c)], respectively. Insets in (e, f) schemati-

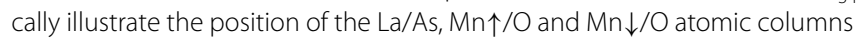

sample (same thickness as the grain used in Fig. 2), for electrons accelerated at $100 \mathrm{kV}$, and with an added aberration $\mathrm{C}_{34}=15 \mu \mathrm{m}$.

Figure 4a shows the calculated the EMCD spectrum image of LaMnAsO emerging from the $\mathrm{Mn}_{3}$ peak in the case of an ideal corrected probe (i.e., the ratio between the magnetic component vs the non-magnetic component in the EEL spectra). Note that at certain individual probe positions, it is possible to detect a non-zero EMCD. However, the intensity is very small (\% 0.05 at the maxima), and its distribution is such that a radially averaged EMCD signal around Mn columns is essentially zero at any distance from the center. The weak EMCD signal, predicted for the case with an ideal corrected probe, is due to broken symmetry, when the beam is off column or, more precisely, off of any mirror symmetry plane. Figure $4 \mathrm{~b}$ shows a calculated EMCD spectrum image obtained using an ideal $\mathrm{C}_{34}$ aberrated probe $\left(C_{34 \mathrm{~b}}=15 \mu \mathrm{m}\right)$. Not only the EMCD signal is significantly stronger, but it also has a well-defined sign reflecting the magnetic moment direction of the $\mathrm{Mn}$ atomic columns. The EMCD signals were also calculated with the experimental probes (corrected and aberrated), and the strength in the EMCD signal was in practical terms the same as the one obtained with the ideal probes.

Figure 5 shows two examples of drift (affine) corrected Z-contrast images and denoised EEL spectra that were acquired simultaneously from $\mathrm{LaMnAsO}$ at a temperature of $22{ }^{\circ} \mathrm{C}(295 \mathrm{~K})$. The data were acquired with the beam along the $c$-axis using the corrected probe and the $\mathrm{C}_{34}=15 \mu \mathrm{m}$ aberrated probe shown in Fig. 2 . The Z-contrast images and the corresponding denoised spectrum images were drift corrected using an affine transformation, as shown in Fig. 6. The spectral data are a subset of 9 different spectrum images containing a total of over 300,000 spectra, with 400 energy channels from 605.0 to $725.0 \mathrm{eV}$. The subset was selected based only on the quality of the corresponding Z-contrast images (i.e., images 


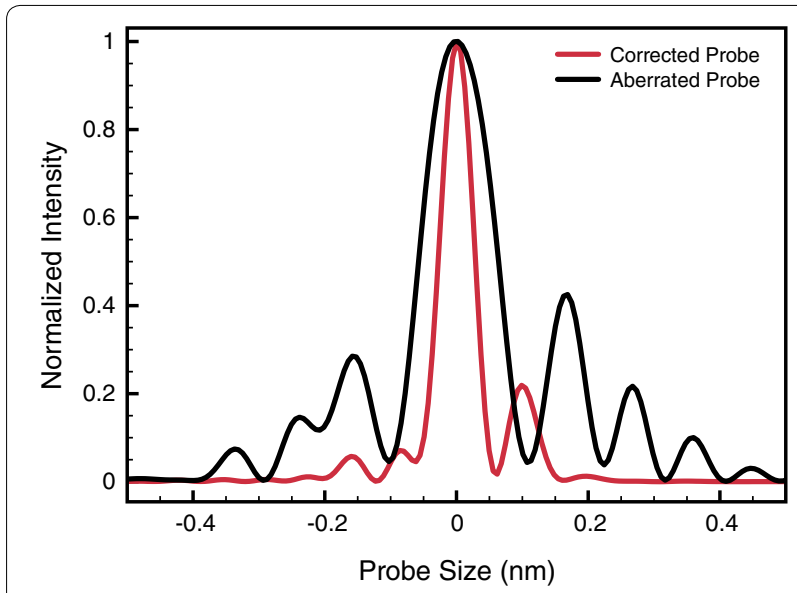

Fig. 3 Electron probe intensity profiles. The electron probes were calculated using the aberrations values tabulated in Table 1 that were obtained from Nion's Ronchigram aberration correction method [10]. The intensity profiles were obtained along the $x$-axis. The corrected probe (red line) corresponds to the intensity profile for the aberrationcorrected electron probe shown in Fig. 2a, while the aberrated probe (black line) is the intensity profile of the $\mathrm{C}_{34}$-aberrated electron probe shown in Fig. 2c

with the smallest spatial drift during data acquisition). The spectra were first normalized to have the same mean value, and aligned in the energy axis via cross-correlation on the La-edge to avoid artifacts (undesired energy shifts) caused by fluctuations on the high tension.

The spectra then were denoised via robust principal component analysis (RPCA) [29, 30] and reconstructed using five components. The number of components was chosen based on the Scree plot (Fig. 7), which displays a clear step after the fifth component. Data reconstruction using 20 components was also performed, but no significant differences to the analysis using only five components were found.

The Mn $\uparrow$ and Mn $\downarrow$ L-edge spectra (shown in Fig. 5b, e) were obtained by averaging all the spectra that are within a radius of $0.08 \mathrm{~nm}$ from the center of all the Mn sites, as shown schematically by the red and blue circles in the Z-contrast images (Fig. 5a, d), respectively. The Mn $\uparrow$ and $\mathrm{Mn} \downarrow$ averaged spectra were obtained only from the affine-corrected data. The $\mathrm{Mn} \uparrow-\mathrm{Mn} \downarrow$ averaged spectra were background corrected using a power-law function (fitting energy region: 606-631 eV) and a non-parametric locally weighted scatterplot smoothing (LOWESS) algorithm [31].

A clear EMCD signature in the EEL spectra, defined as $(\mathrm{Mn} \uparrow-\mathrm{Mn} \downarrow)$ presenting a change of sign in its integrated intensity between the $L_{3}$ and $L_{2}$ peaks, is only visible for the $\mathrm{Mn}$ L-edge acquired using the $\mathrm{C}_{34}$ aberrated probe (Fig. 5e, f). Moreover, the strength of the EMCD signal obtained experimentally at the $\mathrm{Mn} \mathrm{L}_{3}$ peak is comparable with that obtained with the inelastic electron scattering calculations shown in Fig. 4b.

Although LaMnAsO only presents a checkerboard antiferromagnetic ordering at room temperature, different $\mathrm{Mn}$ magnetic orderings were also sought in the spectra to check the robustness of the EMCD signal in the experimental data. No EMCD spectral signature was present for different orderings, as shown in Fig. 8. The spectral difference present in Fig. 8 of a down-up signature on both $L_{3}$ and $L_{2}$ edges is likely caused by slow drift of the high tension tank during the acquisition of the data. The energy drift causes sub-pixel energy shifts $(<0.3 \mathrm{eV})$ for each spectrum within the same spectrum image.
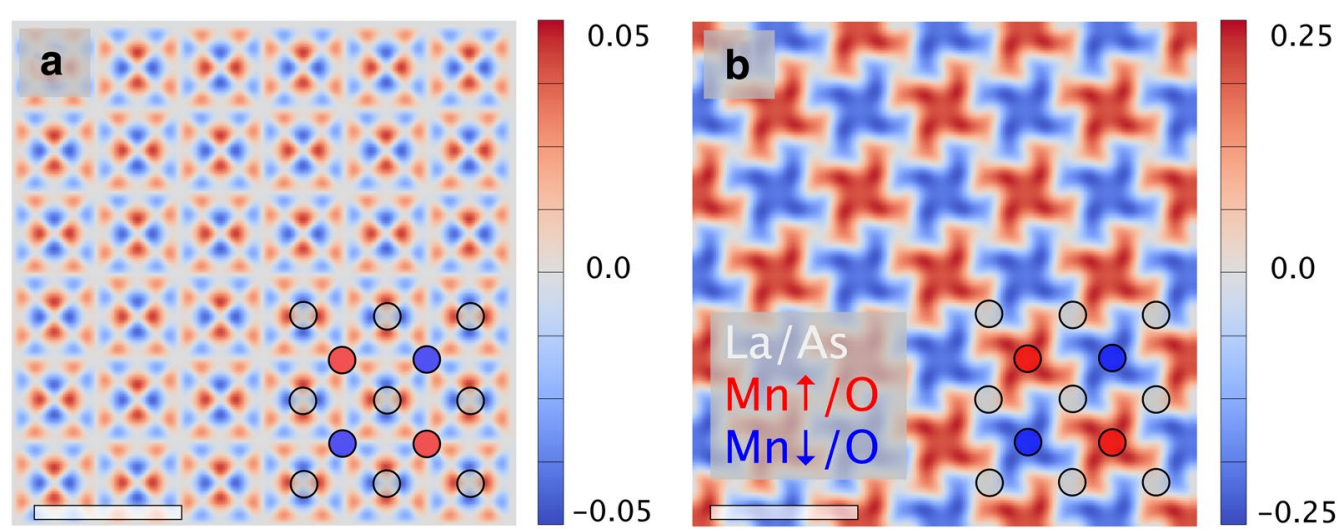

Fig. 4 Calculated energy-loss magnetic circular dichroism spectrum images for the $\mathrm{Mn}_{\mathrm{L}}$ peak using a an ideal corrected and $\mathbf{b}$ an ideal $\mathrm{C}_{34}$-aberrated electron probe $\left(\mathrm{C}_{34 \mathrm{~b}}=15 \mu \mathrm{m}\right)$. Notice that the intensity scale is different in the two panels and that in $(\mathbf{b})$ the $\mathrm{Mn}$ magnetic signal is well defined, with the EMCD signs reflecting the magnetic moment direction of the Mn atomic columns. Insets schematically illustrate the position

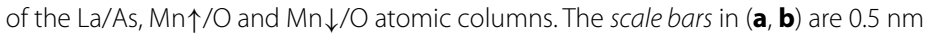




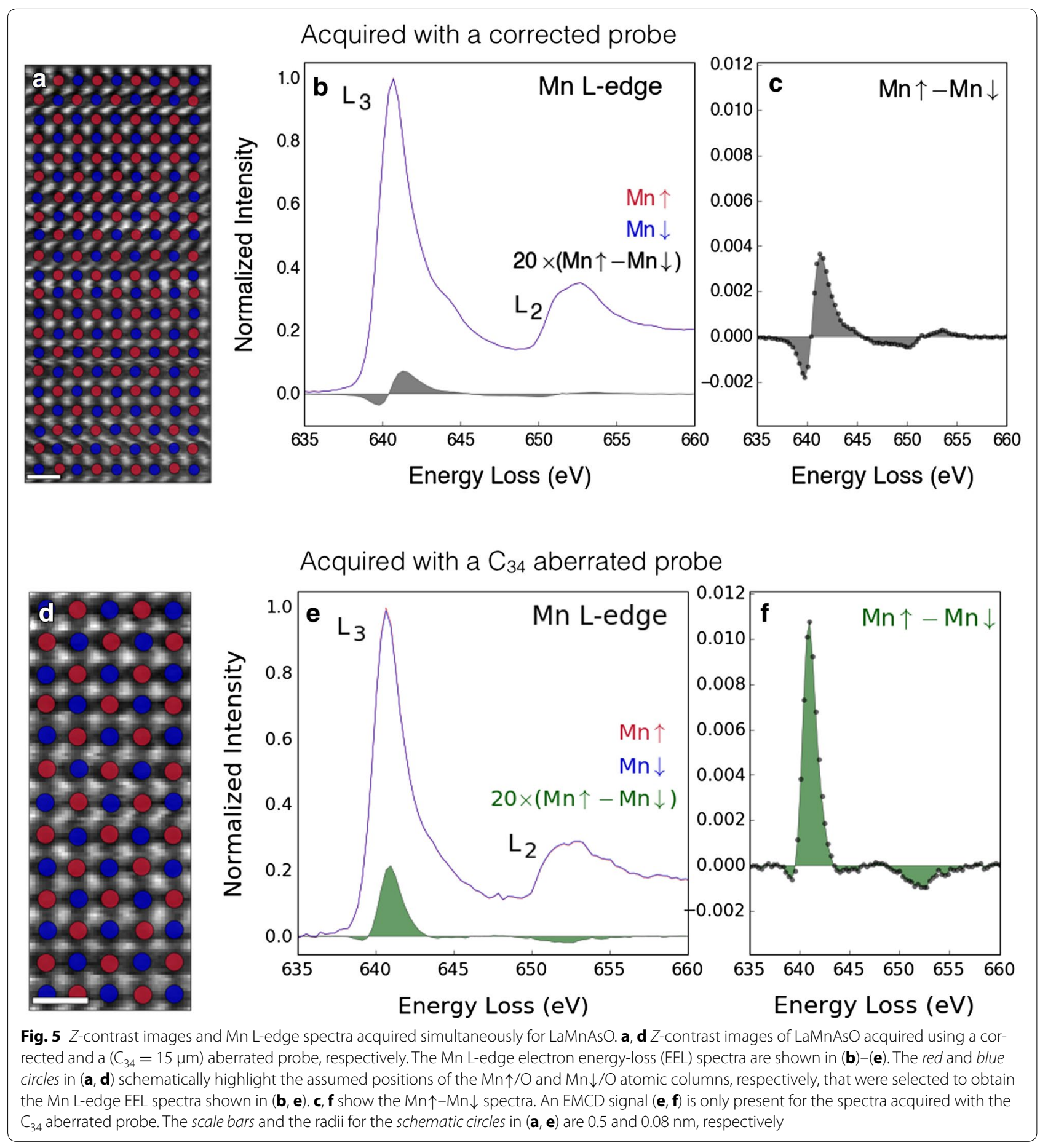

Compared with the checkerboard arrangement, the artifact is enhanced here due to a difference of the average assignment of $\mathrm{Mn}$ atoms with spin up vs spin down in the $\mathrm{y}$-coordinate of the spectrum image.
In principle, the detection of an effective EMCD signal in the EEL spectra also allows us to experimentally try to obtain the Mn orbital/spin moment ratio, $m_{\mathrm{l}} / m_{\mathrm{s}}$, which is not possible to do with XMCD spectroscopy in the case 


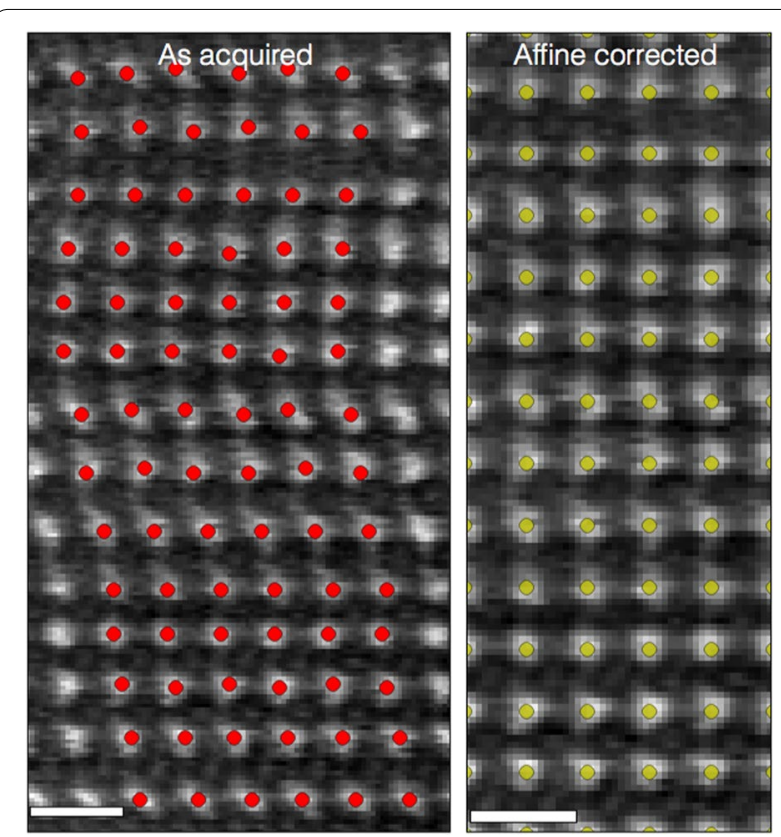

Fig. 6 (Left) Z-contrast image acquired simultaneously with EEL spectra. The red circles show the position of the La atoms that were used for the affine (drift) correction. (Right) The Z-contrast image after performing the affine (drift) transformation. This figure is the same as the one shown in Fig. $5 \mathrm{~d}$. The yellow circles illustrate schematically the positions the La atoms after drift correction. The same affine transformation was applied to the simultaneously acquired spectrum image to reduce drift artifacts. The scale bars are $0.5 \mathrm{~nm}$

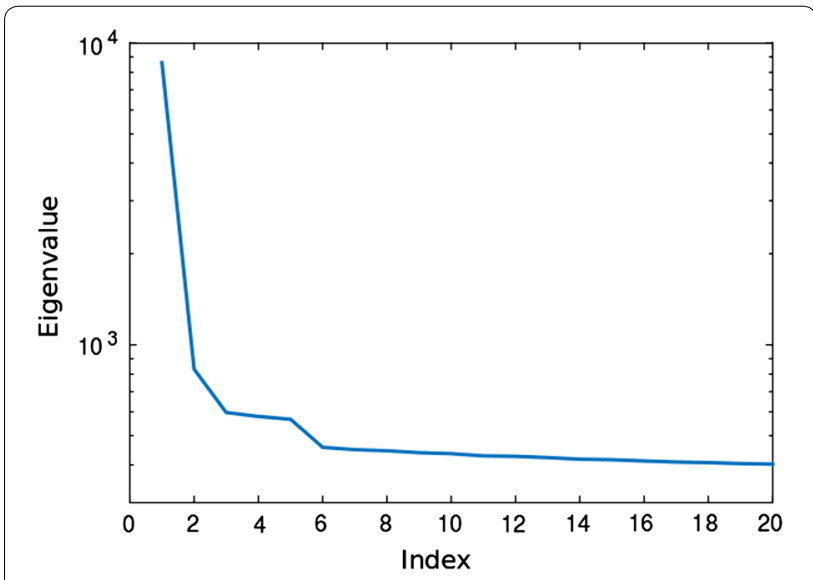

Fig. 7 Scree plot obtained by RPCA on mean-subtracted data. A clear step can be observed after the fifth principal component indicating that the physically relevant information can be described using the first five components in the reconstruction of the EEL spectra of antiferromagnets. The $\mathrm{Mn} m_{\mathrm{l}} / m_{\mathrm{s}}$ ratio was obtained by applying the EMCD sum rules [32,33], such that,

$$
\frac{m_{1}}{m_{\mathrm{s}}}=\frac{2}{3} \frac{\int_{L_{3}} \Delta \mathrm{Mn}(E) \mathrm{d} E+\int_{L_{2}} \Delta \operatorname{Mn}(E) \mathrm{d} E}{\int_{L_{3}} \Delta \operatorname{Mn}(E) \mathrm{d} E-2 \int_{L_{2}} \Delta \operatorname{Mn}(E) \mathrm{d} E},
$$

where $\Delta \mathrm{Mn}$ is the difference between the $\mathrm{Mn} \uparrow$ and $\mathrm{Mn} \downarrow$ spectra. The subscripts $\mathrm{L}_{3}$ and $\mathrm{L}_{2}$ in the integrals refer to the energy integration within each $\mathrm{Mn} \mathrm{L}$ peak. The experimental $\mathrm{Mn} m_{\mathrm{l}} / m_{\mathrm{s}}$ ratio obtained for the EMCD signal is 0.38. First-principles calculations based on density functional theory (DFT) [34, 35] were also performed to obtain an estimate of the $\mathrm{Mn} m_{\mathrm{l}} / m_{\mathrm{s}}$ ratio for the LaMnAsO compound. The DFT calculation gives a Mn $m_{\mathrm{l}} / m_{\mathrm{s}}$ ratio of 0.03 , which is in good agreement with the expected value measured for other $3 d$ transition metals using XMCD [36], but significantly smaller than the experimental EMCD value.

The discrepancy of about an order of magnitude between the experimentally EMCD measured $\mathrm{Mn} m_{\mathrm{l}} / m_{\mathrm{s}}$ ratio and the DFT calculation most likely arises from a quenching of the $\mathrm{Mn} \mathrm{L}_{2}$ peak intensity due to multiple scattering effects and channeling of the electron probe. Theoretical modeling of the EEL spectra needs to be performed to fully understand the origin of the experimentally overestimated $\mathrm{Mn} m_{\mathrm{l}} / m_{\mathrm{s}}$ ratio.

\section{Conclusions}

In this study, the EMCD signal was denoised using robust principal component analysis [29, 30]. However, in the near future, obtaining better EMCD signalto-noise ratios in the EEL spectra should be possible by straightforward improvements in the software controlling the scanning of the electron probe, and by better spectrometers. For instance, the spectra shown in Fig. 5e only utilized $12.5 \%$ of the entire acquired data set per Mn moment orientation (25\% total). The other $75 \%$ of the data is discarded. Since the EMCD is localized in the vicinity of the atomic columns, it makes more sense to use a "selective data acquisition" scheme where only spectra around the center of the atomic columns are collected. This "selective data acquisition" scheme will allow more spectra nearby the center of the atoms to be collected per data set, resulting in a considerable improvement of the EMCD signal-to-noise ratio. Such an improvement would mean that in future EMCD experiments, it will be possible to reveal the magnetic ordering of individual atomic columns and atomic size defects 

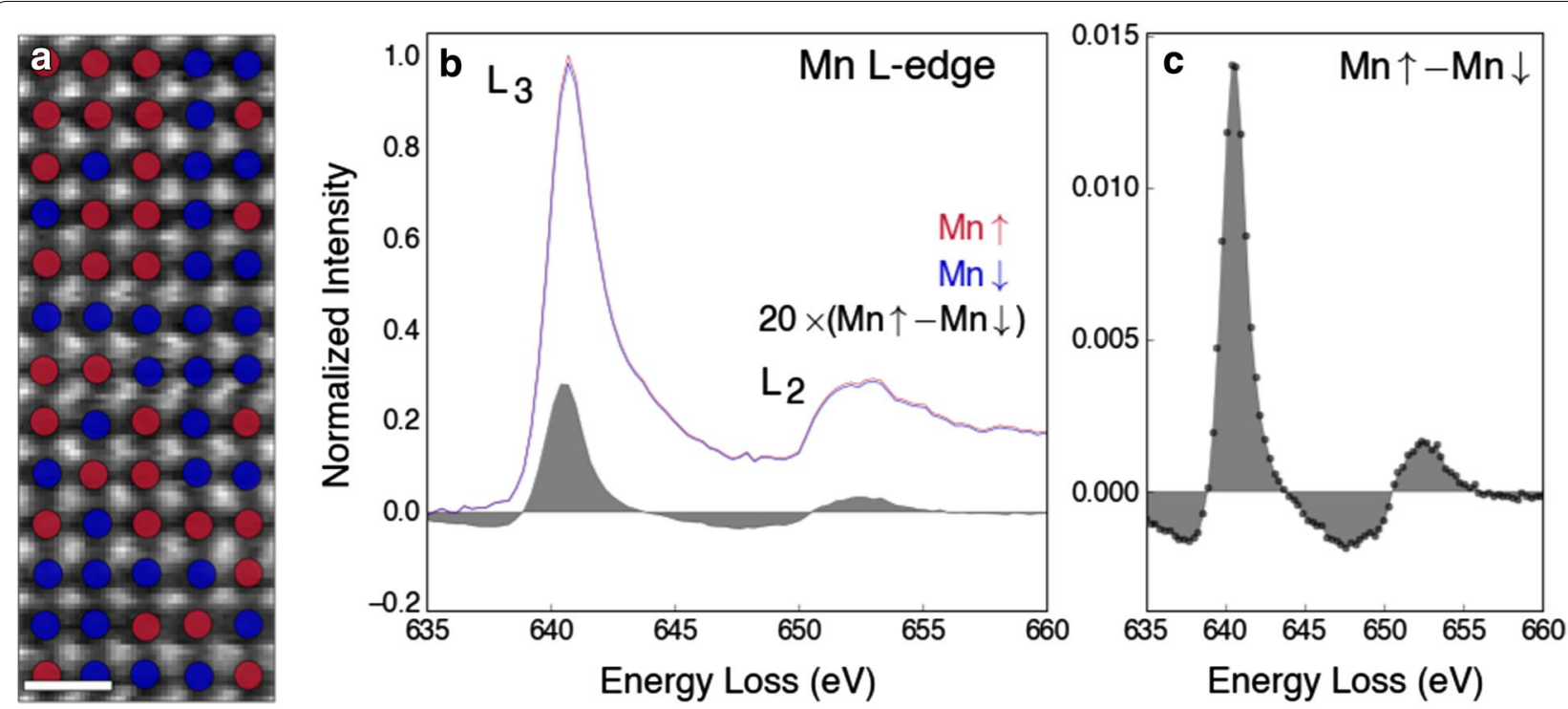

Fig. 8 a Z-contrast image, $\mathbf{b} M n L$-edge, and $\mathbf{c} M n \uparrow-M n \downarrow$ spectra acquired with a $C_{34}$-aberrated probe. The radius used to select the spectra is $0.08 \mathrm{~nm}$, which is the same value as the one used in the main text to obtain the spectra shown in Fig. 5. Only a checkerboard antiferromagnetic ordering is present in LaMnAsO at room temperature. As expected the Mn L-edge spectra do not show an effective EMCD signal when the Mn/O columns are selected in a random ordering (as shown schematically in the Z-contrast image). The scale bar in the Z-contrast image is $0.5 \mathrm{~nm}$

in materials, allowing element-selective quantitative measurements with atomic resolution (and single atom sensitivity) of spin and orbital magnetic moments of ferromagnets, ferrimagnets, and antiferromagnets using EMCD sum rules $[32,33]$. The novel experimental setup presented here could also be used to test candidate magnetic structure models on materials that cannot be made in forms suitable for neutron diffraction.

\footnotetext{
Abbreviations

AMF: around mean-field; DFT: density functional theory; EMCD: energy-loss magnetic circular dichroism; EELS: electron energy-loss spectroscopy; EEL: electron energy-loss; FWHM: full width at half maximum; LSDA: spin polarized local density approximation; RPCA: robust principal component analysis; STEM: scanning transmission electron microscopy; SNR: signal-to-noise ratio; TEM: transmission electron microscopy; XMCD: $X$-ray magnetic circular dichroism; XMLD: X-ray magnetic linear dichroism.
}

\section{Authors' contributions}

$\mathrm{JCl}$ and JR conceived the project and designed the experiment. JCl conducted the STEM and EELS experiments, analyzed the data (with JS and JR) and wrote the paper. JR performed the inelastic electron scattering calculations. MAM synthesized the LaMnAsO sample and performed the magnetic susceptibility measurements. RPCA was performed by JS. Preliminarily statistical analysis was performed by CTS and RRV. CC performed further EELS experiments. ARL helped with the electron optics configuration for the aberrated electron probes. All authors discussed the results and commented on the manuscript. All authors read and approved the final manuscript.

\section{Author details}

${ }^{1}$ Oak Ridge National Laboratory, Center for Nanophase Materials Sciences, Oak Ridge, TN 37831, USA. ${ }^{2}$ Department of Physics and Astronomy, Uppsala University, P.O. Box 516, 75120 Uppsala, Sweden. ${ }^{3}$ Oak Ridge National Laboratory, Materials Science and Technology Division, Oak Ridge, TN 37831, USA.
${ }^{4}$ Oak Ridge National Laboratory, Computational Sciences and Engineering Division, Oak Ridge, TN 37831, USA.

\section{Acknowledgements}

This research was supported by the Center for Nanophase Materials Sciences (CNMS), which is sponsored at Oak Ridge National Laboratory by the Scientific User Facilities Division, Office of Basic Energy Sciences, U.S. Department of Energy (JCl), by the Swedish Research Council, Göran Gustafsson Foundation and Swedish National Infrastructure for Computing (NSC center) (JR), and by the Materials Sciences and Engineering Division Office of Basic Energy Sciences, U.S. Department of Energy (MAM, CC, ARL), and by UT-Battelle, LLC, under Contract No. DE-AC05-00OR22725 with the U.S. Department of Energy (CTS, RRV). Helpful discussions on correlated noise with B. Violinist and T. Kuula, on EELS data analysis and EMCD with Maria Varela, Wu Zhou and Kristiaan Pelckmans, and on setting up aberrated probes in the electron microscope with Niklas Dellby are gratefully acknowledged. The authors would like to specially thank Ondrej Krivanek and Niklas Dellby for discussions on electron microscopy, and for introducing the authors (JCl, JR, and ARL) during Nion's organized Swift Workshop in March 2014. Without their intervention this manuscript would not have been possible.

\section{Competing interests}

The authors declare that they have no competing interests.

Received: 28 January 2016 Accepted: 9 May 2016

Published online: 27 May 2016

\section{References}

1. van der Laan, G., Thole, B.T.: Strong magnetic $x$-ray dichroism in $2 p$ absorption spectra of $3 d$ transition-metal ions. Phys. Rev. B 43, 13401 (1991)

2. Schütz, G., et al.: Absorption of circularly polarized $x$ rays in iron. Phys. Rev. Lett. 58, 737 (1987)

3. Schattschneider, P., et al.: Detection of magnetic circular dichroism using a transmission electron microscope. Nature 441, 486 (2006) 
4. Salafranca, J., et al.: Surfactant organic molecules restore magnetism in metal-oxide nanoparticles surfaces. Nano Lett. 12, 2499 (2012)

5. Schattschneider, P., et al.: Detection of magnetic circular dichroism on the two-nanometer scale. Phys. Rev. B 78, 104413 (2008)

6. Batson, P.E., Dellby, N., Krivanek, O.L.: Sub-ångstrom resolution using aberration corrected electron optics. Nature $\mathbf{4 1 9}, 94$ (2002)

7. Varela, M., et al.: Spectroscopic imaging of single atoms within a bulk solid. Phys. Rev. Lett. 92, 095502 (2004)

8. Muller, D., et al.: Atomic-scale chemical imaging of composition and bonding by aberration-corrected microscopy. Science 319, 1073 (2008)

9. Rusz, J., Idrobo, J.C., Bhowmick, S.: Achieving atomic resolution magnetic dichroism by controlling the phase symmetry of an electron probe. Phys. Rev. Lett. 113, 145501 (2014)

10. Krivanek, O.L., Dellby, N., Lupini, A.: Towards sub-Å electron beams. Ultramicroscopy 78, 1 (1999)

11. Haider, M., Mueller, H., Uhlemann, S.: Present and future hexapole aberration correctors for high-resolution electron microscopy. Adv. Imag. Electron. Phys. 153, 43-119 (2008)

12. Kamihara, Y., Watanabe, T., Hirano, M., Hosono, H.: Iron-based layered superconductor $\left.\mathrm{La} \mathrm{O}_{1}-{ }_{x} \mathrm{~F}\right] \mathrm{FeAs}(x=0.05-0.12)$ with $T_{c}=26 \mathrm{~K}$. J. Am. Chem. Soc. 130, 3296 (2008)

13. Emery, N., et al:: Giant magnetoresistance in oxypnictides (La,Nd)OMnAs. Chem. Commun. 46, 6777 (2010)

14. McGuire, M.A., et al.: Short-and long-range magnetic order in LaMnAsO. Phys. Rev B. 93, 054404 (2016)

15. Sun, Y.-L., et al.: Insulator-to-metal transition and large thermoelectric effect in $\mathrm{La}_{1}{ }_{-} \mathrm{Sr}_{x} \mathrm{MnAsO}$. EPL 98, 17009 (2012)

16. Rusz, J., Bhowmick, S., Eriksson, M., Karlsson, N.: Scattering of electron vortex beams on a magnetic crystal: towards atomic-resolution magnetic measurements. Phys. Rev. B. 89, 134428 (2014)

17. Rusz, J., Rubino, S., Eriksson, O., Oppeneer, P.M., Leifer, K.: Local electronic structure information contained in energy-filtered diffraction patterns. Phys. Rev. B 84, 064444 (2011)

18. Krivanek, O.L., et al.: An electron microscope for the aberration-corrected era. Ultramicroscopy 108, 179 (2008)

19. Egerton, R.F.: Electron energy-loss spectroscopy in the electron microscope. Springer Science + Business Media, Berlin (2011)
20. Egerton, R.F., Li, P., Malac, M.: Radiation damage in the TEM and SEM. Micron 35, 399 (2004)

21. Rusz, J., Idrobo, J.C.: Aberrated electron probes for magnetic spectroscopy with atomic resolution: theory and practical aspects. Phys. Rev. B. 93 , 104420 (2016)

22. Blaha, P., Schwarz, K., Madsen, G., Kvasnicka, D., Luitz, J.: WIEN2k, an augmented plane wave+local orbitals program for calculating crystal properties. Karlheinz Schwarz, Techn. Universität Wien, Austria (2001)

23. Czyzyk, M.T., Sawatzky, G.A.: Local-density functional and on-site correlations: the electronic structure of $\mathrm{La}_{2} \mathrm{CuO}_{4}$ and $\mathrm{LaCuO}_{3}$. Phys. Rev. B 49, $14211(1994)$

24. Uchida, M., Tonomura, A.: Generation of electron beams carrying orbital angular momentum. Nature 464, 737 (2010)

25. Verbeeck, J., Tian, H., Schattschneider, P.: Production and application of electron vortex beams. Nature 467, 301 (2010)

26. McMorran, B.J., et al.: Electron vortex beams with high quanta of orbital angular momentum. Science 331, 192 (2011)

27. Verbeeck, J., Tian, H., Béché, A.: A new way of producing electron vortex probes for STEM. Ultramicroscopy 113, 83 (2012)

28. Oxley, M.P., Cosgriff, E.C., Allen, L.J.: Nonlocality in imaging. Phys. Rev. Lett. 94, 203906 (2005)

29. Hubert, M., Rousseeuw, P.J., Branden, K.V.: ROBPCA: a new approach to robust principal component analysis. Technometrics $\mathbf{4 7}, 64$ (2005)

30. Verboven, S., Hubert, M.: MATLAB library LIBRA. Comp. Stat. 2, 4 (2010)

31. Cleveland, W.S.: Robust locally weighted regression and smoothing scatterplots. J. Am. Stat. Assoc. 74, 829 (1979)

32. Rusz, J., Eriksson, O., Novak, P., Oppeneer, P.M.: Sum-rules for electron energy-loss near-edge spectra. Phys. Rev. B 76, 060408(R) (2007)

33. Calmels, L., et al.: Experimental application of sum rules for electron energy loss magnetic chiral dichroism. Phys. Rev. B 76, 060409(R) (2007)

34. Hohenberg, P., Kohn, W.: Inhomogeneous electron gas. Phys. Rev. 136 B864 (1964)

35. Kohn, W., Sham, L.J.: Self-consistent equations including exchange and correlation effects. Phys. Rev. 140, A1133 (1965)

36. Chen, C.T., et al.: Experimental confirmation of the X-ray magnetic circular dichroism sum rules for iron and cobalt. Phys. Rev. Lett. 75, 152 (1995)

\section{Submit your manuscript to a SpringerOpen ${ }^{\circ}$ journal and benefit from:}

- Convenient online submission

- Rigorous peer review

- Immediate publication on acceptance

- Open access: articles freely available online

- High visibility within the field

- Retaining the copyright to your article

Submit your next manuscript at $\boldsymbol{\nabla}$ springeropen.com 